\title{
Bow Hunter's Stroke Associated with an Aberrant Course of the Vertebral Artery
}

\author{
-Case Report- \\ Satoru ShIMIZU, Masaru YAMAdA, Hiroshi TAKAgI, Kiyotaka FuJII*, \\ and Shinichi KAN** \\ Department of Neurosurgery, Yamato City Hospital, Yamato, Kanagawa; Departments \\ of *Neurosurgery and ${ }^{* *}$ Radiology, Kitasato University School \\ of Medicine, Sagamihara, Kanagawa
}

\begin{abstract}
A 53-year-old male presented with repeated vertebrobasilar insufficiency on turning the head to the left. Angiography revealed severe stenosis of the dominant right vertebral artery at the atlantoaxial level in this position. Decompression surgery for the affected vertebral artery at the transverse foramen of the atlas was planned. However, surgery revealed an aberrant course of the artery, turning at the orifice of the transverse foramen of the atlas and perforating the dura at the occipitoatlantal level after passing through the bony canal of the atlas. Therefore, decompression was performed at the bony canal, which was the contributing site, and the symptoms improved. Bow hunter's stroke may be caused by atlantoaxial arterial anomalies, so accurate preoperative evaluation of the region is necessary to avoid anatomical confusion at surgery.
\end{abstract}

Key words: bow hunter's stroke, decompression surgery, vertebral artery, anomaly

\section{Introduction}

Stenotic change of the vertebral artery (VA) can occur at the atlantoaxial level when the head is turned to the opposite side. This vascular disorder is named "bow hunter's stroke." D] Decompression of the affected VA at the atlantoaxial portion has been proposed as a surgical treatment for bow hunter's stroke. $^{2-4,6)}$ Preoperative assessment of the affected site is indispensable for successful decompression surgery. We describe a case of bow hunter's stroke in which preoperative investigations failed to detect the abnormal anatomical relationship between the VA and the atlas.

\section{Case Report}

A 53-year-old male complained of repeated vertigo and fainting within a few seconds of turning his head to the left over a period of 6 months. Physical examination found no other abnormality. Cervical radiography showed no abnormality of the dynamics at the atlantoaxial joint and angiography revealed no abnormality of the dominant right VA in the neutral position (Fig. $1 \mathrm{left}$ ). However, severe stenosis occurred at the atlantoaxial level on turning the head 50 degrees to the left (Fig. 1 right). The left VA was hypoplastic. Axial computed tomography (CT) of the atlas revealed normal image (Fig. 2).

Decompression of the affected VA at the transverse foramen of the atlas was planned, but surgical exposure revealed an abnormal course of the VA at the level of the atlas. The VA turned at the orifice of the transverse foramen, and perforated the dura at the occipitoatlantal level after passing the bony canal of the atlas (Fig. 3). The foramen was recognizable as normal size, and filled with soft tissue but without vascular structures. No other anomaly of the artery was found. Therefore, the bony canal was drilled out for decompression of the VA and the

Received May 6, 1999; Accepted August 2, 1999

Author's present address: S. Shimizu, M.D., Department of Neurosurgery, Kitasato University School of Medicine, Sagamihara, Kanagawa, Japan. 

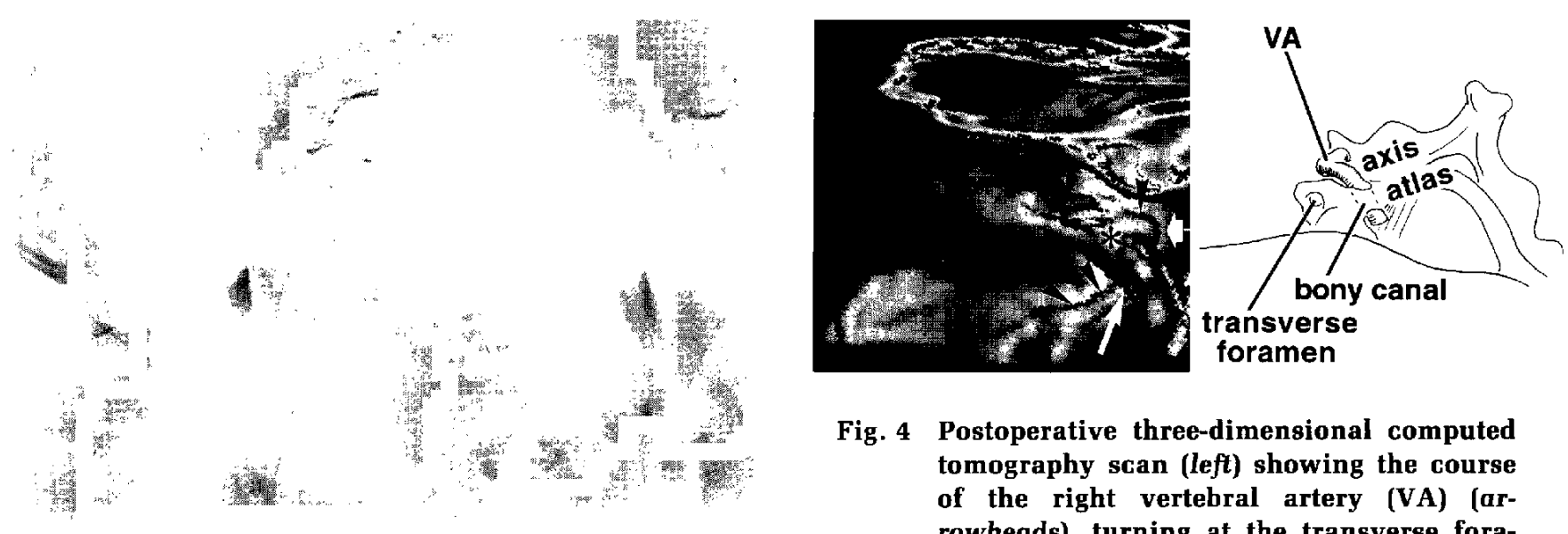

Fig. 1 Lateral right vertebral angiograms, in the neutral position showing no abnormality of the course of the artery (left), and with head rotation 50 degrees to the left showing severe stenosis at the atlantoaxial level (right, arrow).

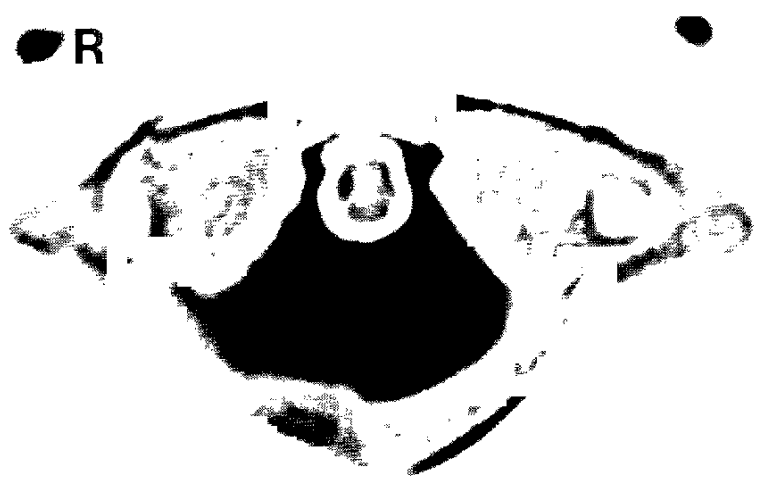

Fig. 4 Postoperative three-dimensional computed tomography scan (left) showing the course of the right vertebral artery (VA) (arrowheads), turning at the transverse foramen of the atlas (arrow) and entering the dura at the occipitoatlantal level (thick arrow). The bony canal of the atlas is drilled (asterisk). Schematic drawing (right) showing the aberrant course of the VA, turning at the caudal site of the transverse foramen of the atlas and perforating the dura at the occipitoatlantal level after passing through the bony canal.

symptoms improved. Postoperative three-dimensional (3D) CT revealed these abnormal anatomical features (Fig. 4).

\section{Discussion}

In the present case, preoperative angiography showed no aberrant course of the VA at the atlantoaxial level, so decompression was planned at the transverse foramen of the atlas, where the VA generally perforates. ${ }^{1]}$ However, the VA turned at the caudal site of the foramen and perforated the dura at the occipitoatlantal level after passing through the bony canal of the atlas. This unexpected anomaly may be recognized as a variant of the $\mathrm{C}-2$ segmental type of VA. ${ }^{5.8)}$ The C-2 segmental type of
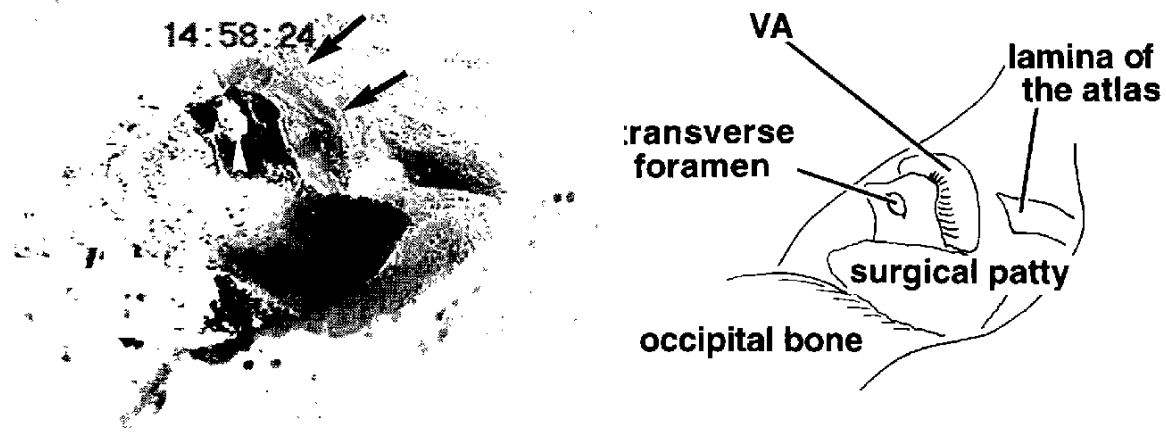

Fig. 3 Operative photograph, craniocaudal view (left), and schematic diagram (right), showing the aberrant course of the vertebral artery (VA) at the level of the atlas. The VA is exposed in the drilled bony canal of the atlas [arrows] and does not perforate the transverse foramen (arrowhead). 
VA includes intraspinal coursing after leaving the transverse foramen of the axis without passing through the transverse foramen of the atlas. The incidence has been $0.6 \%$ to $0.7 \%$ in large angiographical studies. The embryological origin is a connection between the intersegmental artery as the origin of the VA and the second dorsal branch running along the C-2 spinal nerve root during the second fetal stage (fetal length 5 to $6 \mathrm{~mm}$ ). ${ }^{5,8)}$ In contrast, the VA ran into the spinal canal at a higher site associated with the bony canal of the atlas in the present patient. The transverse foramen of the atlas, which acts as a tethering site of the VA in bow hunter's stroke, ${ }^{2-4,6]}$ was not affected. Possibly tethering and stretching of the artery occurred due to the anomalous course in our patient. This configuration has not been categorized among the previously described atlantoaxial arterial anomalies. ${ }^{5,8)}$ Clearly, this possibility should be taken into account when decompression surgery for this condition is planned. Digital subtraction angiography does not allow evaluation of anatomical relationships between vessels and bony structures, so preoperative 3D CT is recommended to obtain accurate anatomical information sufficient to detect unexpected courses of arteries.

\section{References}

1) de Oliveira E, Rhoton AL Jr, Peace D: Microsurgical anatomy of the region of the foramen magnum. Surg Neurol 24: 293-352, 1985

2) Fox MW, Piepgras DG, Bartleson JD: Anterolateral decompression of the atlantoaxial vertebral artery for symptomatic positional occlusion of the vertebral artery. Case report. J Neurosurg 83: 737-740, 1995

3) Hanakita J, Miyake $H$, Nagayasu $S$, Nishi $S$, Suzuki T: Angiographic examination and surgical treatment of bow hunter's stroke. Neurosurgery 23: 228-232, 1988

4) Matsuyama T, Morimoto T, Sakaki T: Comparison of C1-2 posterior fusion and decompression of the vertebral artery in the treatment of bow hunter's stroke. J Neurosurg 86: 619-623, 1997

5) Sato K, Watanabe T, Yoshimoto T, Kameyama M: Magnetic resonance imaging of C2 segmental type of vertebral artery. Surg Neurol 41: 45-51, 1994

6) Shimizu T, Waga S, Kojima T, Niwa S: Decompression of the vertebral artery for bow-hunter's stroke. Case report. J Neurosurg 69: 127-131, 1988

7) Sorensen BF: Bow hunter's stroke. Neurosurgery 2: 259-261, 1978

8) Tokuda $K$, Miyasaka $K$, Abe $H$, Abe $S$, Takei $H$, Sugimoto $S$, Tsuru M: Anomalous atlantoaxial portions of vertebral and posterior inferior cerebellar arteries. Neuroradiology 27: 410-413, 1985

Address reprint requests to: S. Shimizu, M.D., Department of Neurosurgery, Kitasato University School of Medicine, 1-15-1 Kitasato, Sagamihara, Kanagawa 228-8555, Japan. 